\title{
Application of $\mathrm{CD}$ and $\mathrm{Eu}^{3+}$ dual emission MOF colorimetric fluorescent probe based on neural network in $\mathrm{Fe}^{3+}$ detection
}

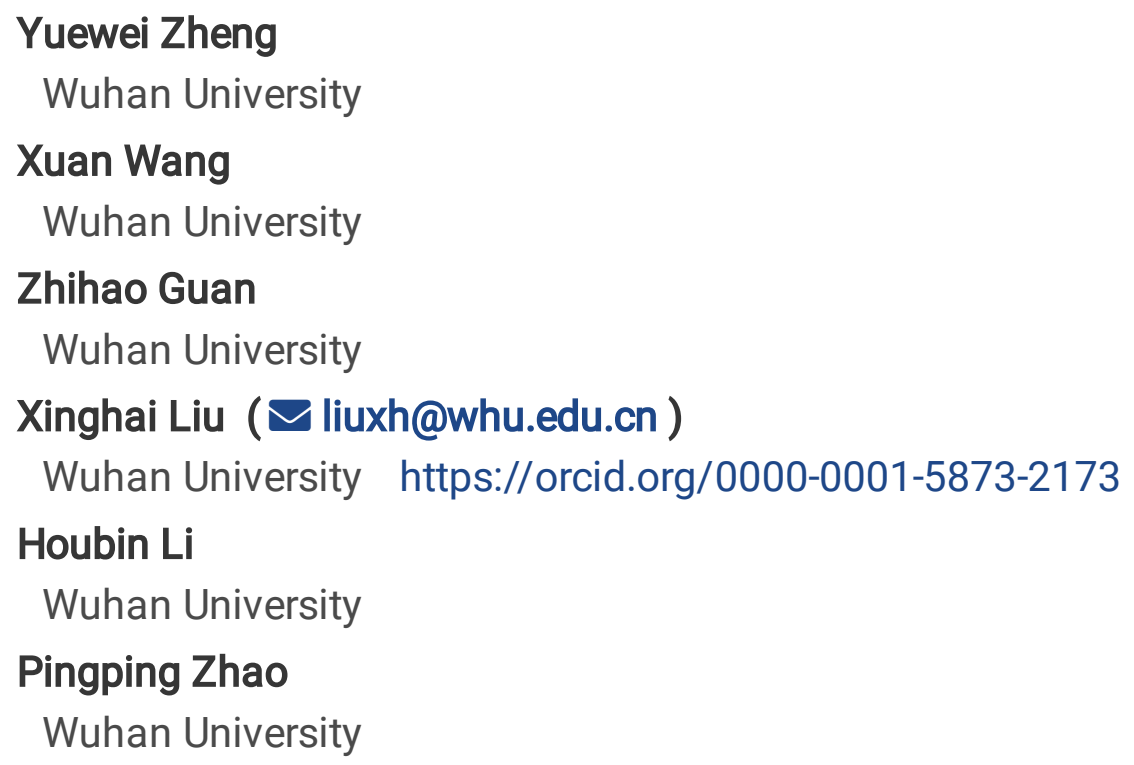

Yuewei Zheng

Wuhan University

Xuan Wang

Wuhan University

Zhihao Guan

Wuhan University

Xinghai Liu ( $\square$ liuxh@whu.edu.cn )

Wuhan University https://orcid.org/0000-0001-5873-2173

Houbin Li

Wuhan University

Pingping Zhao

Wuhan University

\section{Research Article}

Keywords: Carbon dots, MOF, Ion detection, Neural network

Posted Date: February 7th, 2022

DOI: https://doi.org/10.21203/rs.3.rs-1311338/v1

License: (c) (i) This work is licensed under a Creative Commons Attribution 4.0 International License. Read Full License 


\section{Abstract}

A new highly fluorescent hybrid material (CD@Eu-MOF) was synthesized by encapsulating CD (Carbon Dots) prepared from citric acid and ethylenediamine on the basis of a metal-organic framework prepared from $\mathrm{Eu}^{3+}$ and 1,2,4-benzenetricarboxylic acid. The prepared composite not only maintains the excellent fluorescence properties of $\mathrm{CD}$ and $\mathrm{Eu}^{3+}$ respectively, but also forms a dual-emission fluorescence system, and the system has good stability in aqueous solution. It was further used as a novel fluorescent probe for the detection of $\mathrm{Fe}^{3+}$, which can effectively exclude the interference of other metal ions from the detection, and the intensity ratio of $I_{E u} / I_{C D}$ of $C D @ E u-M O F$ material has a good linear relationship with $\mathrm{Fe}^{3+}$ in the range of 1-200 $\mu \mathrm{M}$. In this study, computer vision and BP (Back Propagation) neural network were used to train and fit the sample data, and it was verified that the actual fluorescence color of $C D$ has a good linear relationship with $\mathrm{Fe}^{3+}$ concentration. In addition, the BP neural network also verified that the fluorescence spectrum data of CD@Eu-MOF also has a good linear relationship with $\mathrm{Fe}^{3+}$ concentration. This study provides a new method for the fabrication of ratio and colorimetric $\mathrm{Fe}^{3+}$ fluorescence sensors, and provides a feasible solution for the simple and visual detection of $\mathrm{Fe}^{3+}$ in the environment.

\section{Introduction}

$\mathrm{Fe}^{3+}$ plays a crucial role in all essential metabolic processes in nature, including the metabolism of oxygen and the catalysis of biological enzymes.[1] $\mathrm{Fe}^{3+}$ is widely distributed in our environment, and its determination is therefore of great importance for human health. The existing detection schemes for small molecules including $\mathrm{Fe}^{3+}$ include Raman spectroscopy, Fourier transform infrared spectroscopy, liquid chromatography-mass spectrometry and a series of methods with the help of modern instruments. [2-4] Although these methods have good upper and lower detection limits, they greatly increase the detection time due to the more complicated sampling and preparation process. Therefore, it is important to develop a fast and efficient method for the detection of $\mathrm{Fe}^{3+}$. The fluorescence assay is the best choice with the above advantages.[5-9]

Among them, carbon quantum dots are the best choice because of their superior fluorescence properties and high specificity for detection.[6] In recent years, carbon quantum dots have been successfully used for the detection of some specific substances because of their microscopic size, tunability of surface functionality, as well as their low toxicity and abundant synthesis methods.[10,11] This includes small molecules such as glucose, dopamine, isoleucine enantiomers, antibiotics and malachite green as well as bacteria such as E. coli.[12-17] With further research, carbon quantum dots have also been gradually used for the detection of metal ions, including $\mathrm{Hg}^{2+}, \mathrm{Ag}^{+}$, and $\mathrm{Cr}^{6+}$.[18-21] Different kinds of carbon quantum dots can specifically recognize different metal ions because of their abundant groups on the surface, which provides the possibility of detection.[22] However, the detection of metal ions by a single carbon quantum dot is limited, and we improved its detection capability and range by introducing another structure with fluorescence to combine with carbon quantum dots to become a colorimetric fluorescence 
sensor.[21-23] Currently, this colorimetric fluorescent sensor has been successfully implemented to detect substances such as urea, ascorbic acid, hypochlorous acid, cephalexin and even proteins.[24-28]

We chose metal organic frameworks (MOF) synthesized from rare earth elements as another system for fluorescence generation. Because of the porous structure and some unique optical properties of MOF, it enables them to be used not only for gas storage and catalysis, but also for recognition and sensing of ions and molecules and biomolecules.[29-31] Meanwhile, the rare earth element Eu has the ability to fluorescence luminescence due to its unique electronic orbitals.[32] Thus, MOF through the combination

of $\mathrm{Eu}^{3+}$ and 1,2,4-benzenetricarboxylic acid thus have the fluorescent system of the element Eu. This MOF was found to be sensitive to $\mathrm{Hg}^{2+}$ by experiments. By introducing $\mathrm{CD}$ into this MOF, we were able to combine the advantages of both and improve their detection ability under various complex environments.

Computer vision is the simulation of biological vision using computers and related devices. Its main task is to obtain three-dimensional information about the corresponding scene by processing a captured picture or video. It is due to the limitations of human's own naked eye to discriminate colors that computer vision was introduced to distinguish colors that are difficult to distinguish with human eyes and to identify RGB values of colors. The detection of curcumin and hypochlorite, acetylcholinesterase, cephalexin and parasites in drinking water was achieved by using computer vision for fluorescence acquisition.[26, 27, 33, 34] The recognition and acquisition of fluorescence by computer vision not only improved the recognition speed, but also greatly increased the upper and lower limits of recognition and the accuracy of recognition. Rapid and accurate detection of fluorescent probes became possible.

In this study, we encapsulated CD in a controlled manner in a MOF material with $\mathrm{Eu}^{3+}$ as the metal center, and the resulting CD@Eu-MOF complex contains not only the advantages of lanthanide luminescence of the MOF matrix, but also the characteristic luminescence of $C D$. We found that this novel dual emission is selective for $\mathrm{Fe}^{3+}$. At the same time, we used computer vision to extract the RGB value of the actual fluorescence of $C D$, and trained and predicted the dataset through BP neural network, which verified that the actual fluorescence RGB value of $\mathrm{CD}$ has a good linear relationship with the $\mathrm{Fe}^{3+}$ concentration. In addition, the BP neural network also verified that the fluorescence spectrum data of CD@Eu-MOF also has a good linear relationship with $\mathrm{Fe}^{3+}$ concentration. At present, there are few reports that the MOFencapsulated $\mathrm{CD}$ and $\mathrm{Eu}^{3+}$ are used as fluorescent substances, combined with ratio fluorescent probes, and the detection probe database is formed by computer color recognition, thereby improving the detection accuracy. A feasible solution is provided to further simplify the detection of $\mathrm{Fe}^{3+}$ in the environment.

\section{Experimental}

\subsection{Chemicals and instrumentation}

The drugs used in this experiment were all analytically pure. They can be used without further purification. The drugs were obtained from Sinopharm and Micrel. Anhydrous citric acid and 
ethylenediamine were used for the synthesis of $\mathrm{CD}$. $\mathrm{EuCl}_{3}-6 \mathrm{H}_{2} \mathrm{O}$ and 1,2,4-benzenetricarboxylic acid were used for the synthesis of Eu-MOF. aqueous solutions of $\mathrm{Fe}^{3+}, \mathrm{Cr}^{3+}, \mathrm{Zn}^{2+}, \mathrm{Mn}^{2+}, \mathrm{Cu}^{2+}, \mathrm{K}^{+}, \mathrm{Al}^{3+}, \mathrm{Na}^{+}, \mathrm{Hg}^{2+}$, $\mathrm{Ba}^{2+}, \mathrm{Ca}^{2+}, \mathrm{Pb}^{2+}, \mathrm{Ni}^{2+}, \mathrm{Mg}^{2+}$ were configured from nitrate and chloride salts.

The CD solution with a concentration of $0.06 \mathrm{mg} \mathrm{mL}^{-1}$ was ultrasonically shaken for 5 min to make it well dispersed. The CD solution was then dropped onto the carbon film with a pipette, dried with an infrared baking lamp to evaporate the solvent, and characterized by transmission electron microscope JEM-2100 transmission electron microscope. The samples were prepared by grinding the trace CD powder with dried potassium bromide powder with a mortar, and the CD powder was characterized by Fourier transform infrared spectroscopy using NICLET-5700 to analyze the surface functional groups of CDs. The UV absorption of the CD solution at a concentration of $0.03 \mathrm{mg} \mathrm{mL}^{-1}$ was characterized using a Japanese UV-3600 UV-Vis spectrometer. The wavelength range was set from 200 to $800 \mathrm{~nm}$, and the lattice characteristics of the CDs were characterized by Bruker D8 advance powder diffractometer. The measured $2 \theta$ range was $5^{\circ}-50^{\circ}$ and the scanning speed was $8^{\circ} \mathrm{min}^{-1}$. The excitation and emission spectra of the CD solutions were analyzed using an Edinburgh FS5 fluorescence spectrometer. The light source was a xenon lamp as the light source, the spectral bandwidth was $1.5 \mathrm{~nm}$, the fluorescence emission was recorded in the range of $360-700 \mathrm{~nm}$, and the Dwell time was set to $0.1 \mathrm{~s}$. During the experimental tests, all parameters of the instrument were kept consistent.

\subsection{Synthesis of CD and CD@Eu-MOF}

\subsubsection{Preparation of carbon dots (CD)}

the desired CD were prepared by a one-step hydrothermal synthesis method.[21] The specific steps included weighing anhydrous citric acid $(0.9607 \mathrm{~g})$ and ethylenediamine $(335 \mu \mathrm{L})$, dissolving them in 10 $\mathrm{mL}$ of deionized water and stirring for $30 \mathrm{~min}$. the stirred liquid was transferred to an autoclave $(25 \mathrm{~mL})$ lined with polytetrafluoroethylene and reacted in an oven at $200^{\circ} \mathrm{C}$ for $5 \mathrm{~h}$. After natural cooling to room temperature, the product was centrifuged at $10,000 \mathrm{r} \mathrm{min}^{-1}$ and the supernatant was collected in a dialysis bag (1000 MWCO) for $24 \mathrm{~h}$. Small molecular impurities were removed by this method. Finally, the dialyzed liquid was frozen and finally the $C D$ were prepared by freeze-drying in a freeze-dryer.

\subsubsection{Preparation of CD@Eu-MOF}

The desired Eu-MOF was prepared by hydrothermal synthesis.[22] The procedure was as follows: $\mathrm{EuCl}_{3}{ }^{-}$ $6 \mathrm{H}_{2} \mathrm{O}(0.0366 \mathrm{~g})$ and 1,2,4-benzenetricarboxylic acid $(0.0210 \mathrm{~g})$ were weighed and dissolved in $25 \mathrm{~mL}$ of DMF, then the above-prepared solution of $C D(1 \mathrm{~mL})$ with a concentration of $5 \mathrm{mg} \mathrm{mL}^{-1}$ was added and stirred for $30 \mathrm{~min}$. The stirred liquid was transferred to an autoclave $(50 \mathrm{~mL})$ lined with polytetrafluoroethylene and reacted in an oven at $120^{\circ} \mathrm{C}$ for $24 \mathrm{~h}$. The reaction was carried out at room temperature. After natural cooling to room temperature, the liquid was washed with ethanol three times and centrifuged at $8000 \mathrm{r} \mathrm{min}^{-1}$ for $10 \mathrm{~min}$, and finally dried in a vacuum drying oven at $80^{\circ} \mathrm{C}$ for $8 \mathrm{~h}$ to prepare Eu-MOF. 


\subsection{Stability and selectivity testing}

For pH stability test, $4 \mathrm{mg}$ of CD@Eu-MOF powder was dispersed in $4 \mathrm{~mL}$ of different $\mathrm{pH}$ cache solution and ultrasonically shaken for 5 min to make it well dispersed, and the fluorescence emission data were tested. Storage time stability test, $4 \mathrm{mg}$ of CD@Eu-MOF was dispersed in $4 \mathrm{~mL}$ of deionized water, and its fluorescence emission and XRD data were tested in 1,3, 5, 7 and 9 days consecutively

For the fluorescence test of $\mathrm{Fe}^{3+}, 4 \mathrm{mg}$ of CD@Eu-MOF was dispersed in $4 \mathrm{~mL}$ of different concentrations of $\mathrm{Fe}^{3+}$ solution at room temperature, and then the mixture was shaken thoroughly and made to disperse uniformly by ultrasonication for $5 \mathrm{~min}$. In order to test other metal ions $\left(\mathrm{Cr}^{3+}, \mathrm{Zn}^{2+}, \mathrm{Mn}^{2+}, \mathrm{Cu}^{2+}, \mathrm{K}^{+}, \mathrm{Al}^{3+}\right.$, $\left.\mathrm{Na}^{+}, \mathrm{Hg}^{2+}, \mathrm{Ba}^{2+}, \mathrm{Ca}^{2+}, \mathrm{Pb}^{2+}, \mathrm{Ni}^{2+}, \mathrm{Mg}^{2+}\right)$, the same test procedure as $\mathrm{Fe}^{3+}$ can be used.

\subsection{Fluorescent quenching testing}

Deionized water was used as the reference background, and then 3 mg of Eu-MOF, CD@Eu-MOF and ferric ion-treated CD@Eu-MOF were dispersed in $3 \mathrm{~mL}$ of deionized water and sonicated for 5 min to make them well dispersed, and then the suspension was transferred to a cuvette for testing. For the UV absorption of ferrous ions, it was only necessary to replace Eu-MOF with a solution of $800 \mu \mathrm{mol} \mathrm{L}^{-1}$ of ferrous ions.

\subsection{Prediction model for the concentration of $\mathrm{Fe}^{3+}$}

The fluorescent RGB components of CD solutions with different concentrations of $\mathrm{Fe}^{3+}$ added were collected by computer vision and established correspondence with $\mathrm{Fe}^{3+}$ concentration to establish the prediction function by linear regression algorithm. Meanwhile, a prediction model based on neural network was established for more accurate prediction, and the complex functional relationship between the concentration of $\mathrm{Fe}^{3+}$ and the three RGB components was obtained more accurately by a large amount of data. The relationship between different concentrations of $\mathrm{Fe}^{3+}$ and $\mathrm{CD} @ E$ Eu-MOF fluorescence was directly obtained by using neural network to analyze the CD@Eu-MOF fluorescence spectrum data.

\section{Results And Discussion}

\subsection{Characterization of CD}

The morphology and structure of the CD were determined by transmission electron microscopy (TEM) image analysis using the hydrothermal method. Fig. 1(a) shows the TEM image of the synthesized CD, which shows that they are uniformly dispersed without aggregation, and their particle sizes are in the range of $5-10 \mathrm{~nm}$. By randomly selecting $100 \mathrm{CDs}$ and measuring their particle size, Fig. 1(b), the particle size distribution of the $C D$, shows that the particle size of the carbon dots is about 2-5 nm, and the average particle size is about $3.12 \mathrm{~nm}$. In Fig. 1(a), it can be observed that most of the carbon dots are amorphous carbon particles, and no lattice is observed, and only a very small number of carbon dots can be observed. The XRD pattern of the carbon dots in Fig. 1(c) also confirms the amorphous state of the 
carbon dots, showing that the $\mathrm{CD}$ have a broad peak centered at $25^{\circ}(0.34 \mathrm{~nm})$. The functional groups on the surface of the carbon dots were characterized by FTIR spectroscopy. As shown in Fig. 1(d), the stretching vibration of $\mathrm{C}-\mathrm{OH}$ can be observed at a wave number of $3430 \mathrm{~cm}^{-1}$, and the absorption peaks appearing at $2923 \mathrm{~cm}^{-1}$ and $2850 \mathrm{~cm}^{-1}$ can correspond to the stretching vibration of $\mathrm{C}-\mathrm{H}$. The asymmetric stretching vibrations of $\mathrm{C}-\mathrm{N}$ and $\mathrm{H}-\mathrm{C}$ are present at $1126 \mathrm{~cm}^{-1}$, and the bending vibrations of $\mathrm{N}-\mathrm{H}$ can be observed at $1570 \mathrm{~cm}^{-1}$, as well as the absorption band of $\mathrm{C}=0$ vibrations at $1635 \mathrm{~cm}^{-1}$. From this infrared spectrogram, it can be analyzed that the surface of carbon dots is successfully doped with nitrogen, and the presence of all these functional groups greatly promotes the solubility of carbon dots in water.

\subsection{Characterization of CD@Eu-MOF}

CD were introduced into Eu-MOF by a one-step synthesis method. The generated product is denoted as CD@Eu-MOF. Fig. 2(a) shows the TEM image of CD@Eu-MOF, which shows the tetrahedral lamellar structure, and also shows that there is no obvious aggregation of carbon dots on the outer surface of $\mathrm{MOF}$, indicating that the carbon dots may have entered the framework system of MOF overwhelmingly. Fig. 2(b) shows the XRD patterns of Eu-MOF and CD@Eu-MOF, from which it can be seen that the two spectra are roughly similar, which proves that the addition of carbon dot material to Eu-MOF does not change the crystalline shape of MOF. The characteristic peaks of carbon dots are almost absent in the XRD spectra compared with the strong peaks of MOF, which may be due to the lower concentration of carbon dots or the smaller size of carbon dots compared with the size of MOF base. Fig. 2(c) shows the IR spectra of CD, Eu-MOF and CD@Eu-MOF, the peaks at $3428 \mathrm{~cm}^{-1}$ are the stretching vibration peaks of $\mathrm{O}-\mathrm{H}$ and $\mathrm{N}-\mathrm{H}$ bonds, and the peaks at $2975 \mathrm{~cm}^{-1}$ is the stretching vibration peak of $\mathrm{C}-\mathrm{H}$, the peak at 1654 $\mathrm{cm}^{-1}$ is the stretching vibration peak of $\mathrm{C}=\mathrm{N}$, the peak at $1556 \mathrm{~cm}^{-1}$ is the stretching vibration peak of $\mathrm{C}=0$, the peak at $1386 \mathrm{~cm}^{-1}$ is the stretching vibration peak by $\mathrm{C}=\mathrm{C}$, and the peak at $1051 \mathrm{~cm}^{-1}$ belongs to the stretching vibration peak of C-O. It can be seen that CD@Eu-MOF contains both the characteristic absorption peaks of Eu-MOF and the characteristic peaks of CD, which proves that $C D$ already exists in Eu-MOF. From Fig. 2(d), it can be seen that the electronic spectra of Eu-MOF and CD@Eu-MOF contain the characteristic peaks of Eu, C, and O, but CD@Eu-MOF contains the characteristic peaks of $\mathrm{N}$ elements from carbon dots, which can further indicate the existence and encapsulation of carbon dots in Eu-MOF.

This study also explored the effect of different CD loadings on the fluorescence properties and structural stability of CD@Eu-MOF. CD is the key factor for the detection of $\mathrm{Fe}^{3+}$ in this fluorescent probe. Too low carbon dots will affect the detection performance of $\mathrm{CD} @ E \mathrm{Eu}-\mathrm{MOF}$ for $\mathrm{Fe}^{3+}$, and make the ratio fluorescence transformation insignificant. If it is too high, it may have a certain impact on the structure of the metal organic framework, thereby affecting the fluorescence emission of lanthanide metal ions.[35] In addition, an excessively high loading amount of $C D$ may lead to the aggregation of $C D$, resulting in the self-quenching of $C D$ and reducing the detection performance of $C D$. As shown in Fig. S3, when the loading amount is 5 mg, CD@Eu-MOF has strong CD fluorescence emission, which can have good 
sensing performance for the detected substances. From the regularity of the crystal structure, it is also more excellent. More specific analysis content is in the supporting information.

\subsection{Fluorescence characterization}

The fluorescence spectra of the prepared CD and CD@Eu-MOF were analyzed. As shown in Fig. 3(a), the $\mathrm{CD}$ solution has a clear emission peak at $443 \mathrm{~nm}$ under $340 \mathrm{~nm}$ excitation, which leads to a bright blue fluorescence. The prepared CD@Eu-MOF in the aqueous system showed almost unchanged fluorescence emission from the carbon dots compared to the pure carbon dot solution emission, while a distinct emission peak of Eu-MOF appeared at around 617 nm. As shown in Fig. 3(b), the CD@Eu-MOF solution on the left shows a purple color at $365 \mathrm{~nm}$ UV. Fig. 3(c) shows that the Eu-MOF powder is white in visible light, while Fig. 3(d) shows that the Eu-MOF powder is red in the $365 \mathrm{~nm}$ UV light, which is presumed to be the red fluorescence of Eu in the solid state. It should be noted that the luminescence mechanism of carbon dots is still very complicated and not fully understood, however, there is increasing evidence that the fluorescence emission of carbon dots is related to their surface state.[36] This result shows that the surface state of the carbon dots does not change when they enter the Eu-MOF.

\subsection{Stability Characterization}

The fluorescence stability of CD@Eu-MOF materials is of great importance for their applications. The stability of the material at different $\mathrm{pH}$ values was firstly investigated. The test results are shown in Fig. 4(a). The fluorescence intensity of CD@Eu-MOF is relatively little affected by $\mathrm{pH}$. The XRD patterns of CD@Eu-MOF after different pH treatments were then analyzed, as shown in Fig. 4(b), the XRD data were basically consistent with the original CD@Eu-MOF at pH 4, 7 and 9, so CD@Eu-MOF possessed better fluorescence stability at $\mathrm{pH} 4$ to 9 . Next, the effects of different storage times on the fluorescence of CD@Eu-MOF were analyzed. Fig. 4(c) shows that the fluorescence emission was tested continuously at 1, $3,5,7$, and 9 days, and its fluorescence emission remained stable at 9 days. As shown in Fig. 4(b), the XRD patterns of CD@Eu-MOF were tested after 5 days and 9 days of maintenance in aqueous environment, and it was found that its crystal structure remained intact, which proved that CD@Eu-MOF has good stability in aqueous environment. For the thermal stability analysis as shown in Fig. 4(d), it can be obtained that the thermal stability of Eu-MOF is better than that of single CD. for Eu-MOF, the mass change of Eu-MOF is not significant in the range of $40-200^{\circ} \mathrm{C}$ with a decrease in mass ratio of $9 \%$, which corresponds to the loss of very little water as well as DMF in the MOF structure. In the $200-350^{\circ} \mathrm{C}$ range, the mass of Eu-MOF decreases rapidly, with a mass loss of $11 \%$ occurring, a stage corresponding to the disintegration of some organic ligands. In the interval of $350-600^{\circ} \mathrm{C}$, the mass of Eu-MOF decreases significantly, and the mass after warming is about $60 \%$ of the original mass; this stage may correspond to the removal of the remaining organic fraction. the final form after $600^{\circ} \mathrm{C}$ corresponds to the amorphous europium oxide. The thermal stability of CD@Eu-MOF is similar to that of Eu-MOF when CD is loaded onto Eu-MOF, indicating that the loading of CD does not affect the thermal stability of MOF.

\subsection{Selective Characterization}


Since CD is an important component of colorimetric fluorescent probes, the ability of a single CD to detect metal ions such as $\mathrm{Fe}^{3+}$ was investigated. According to Fig. S1, the fluorescence of CD has a good linear response relationship with $\mathrm{Fe}^{3+}$ concentration. At the same time, in the detection of various metal ions, $\mathrm{Fe}^{3+}$ has the best quenching effect on $\mathrm{CD}$ and good anti-interference performance. In order to further verify the relationship between the fluorescence of $C D$ and $\mathrm{Fe}^{3+}$ concentration, the RGB values corresponding to $\mathrm{CD}$ under different $\mathrm{Fe}^{3+}$ concentrations were collected by computer vision, and the $\mathrm{BP}$ neural network was used to analyze and predict. As shown in Fig. S2 and Table S1, the detection accuracy after training reaches $99.64 \%$. All of these demonstrate the feasibility of constructing colorimetric fluorescent probes using CD. More specific analysis content is in the supporting information.

After completing the specificity and selectivity analysis of $\mathrm{CD}$ for $\mathrm{Fe}^{3+}$ detection, we next analyzed the effect of CD@Eu-MOF on the specificity and selectivity of Fe ${ }^{3+}$ detection. As shown in Fig. 5(a), the fluorescence of CD@Eu-MOF at 443 nm gradually decreased with the gradual increase of the concentration of $\mathrm{Fe}^{3+}$ in the range of $0-200 \mu \mathrm{M}$, while the fluorescence intensity at $617 \mathrm{~nm}$ remained relatively constant, which proved that $\mathrm{CD} @ E \mathrm{E}-\mathrm{MOF}$ is an ideal ratio metric fluorescent material for the detection of $\mathrm{Fe}^{3+}$. The intensity ratio of $I_{E u} / I_{C D}$ of $\mathrm{CD} @ E$ Eu-MOF material has a good linear relationship with $\mathrm{Fe}^{3+}$ within $0-200 \mu \mathrm{M}$, as shown in Fig. $5(\mathrm{~b})$, and the calculated $\angle O D$ of the material is about $0.91 \mu \mathrm{M}$ and $\mathrm{R}^{2}$ is about 0.997 at a signal-to-noise ratio of 3, while the EPA (Environmental Protection Agency) allows the maximum concentration of $\mathrm{Fe}^{3+}$ in drinking water to be $5000 \mu \mathrm{M}$, and the detection limit of the material is much lower than this requirement. BP neural network is used to train and analyze the spectral data of CD@Eu-MOF. The input layer is the fluorescence intensity ratio of $I_{E u}$ to $I_{C D}$, the number of hidden layers is set to 10 , and the output layer is the concentration of $\mathrm{Fe}^{3+}$. Training set: test set: verification set is $7: 3: 1$. The prediction accuracy after training reached $99.38 \%$. The model is used to predict and verify the $\mathrm{Fe}^{3+}$ concentration, and the results are shown in Table 1.

Table $1 \mathrm{Fe}^{3+}$ concentration prediction (CD@Eu-MOF) 


\begin{tabular}{|llll|}
\hline Sample & Actual Concentration & Predicted Concentration & Result \\
\hline 01 & 10.0 & 9.9 & YES \\
\hline 02 & 30.0 & 30.2 & YES \\
\hline 03 & 45.0 & 44.7 & YES \\
\hline 04 & 60.0 & 58.9 & YES \\
\hline 05 & 85.0 & 85.7 & YES \\
\hline 06 & 100.0 & 99.4 & YES \\
\hline 07 & 120.0 & 120.2 & YES \\
\hline 08 & 155.0 & 149.8 & NO \\
\hline 09 & 185.0 & 186.4 & YES \\
\hline
\end{tabular}

In addition, the selectivity of CD@Eu-MOF for $\mathrm{Fe}^{3+}$ detection was also analyzed, and a series of metal ions that may interfere with CD@Eu-MOF were selected as interference terms. As shown in Fig. 5(c), the $I_{E u} / I_{C D}$ fluorescence intensity ratio in CD@Eu-MOF basically did not change much when other metal ions were added. As shown in Fig. 5(d), the color of CD@Eu-MOF is purple when no iron ion is added. And when iron ions are added, as shown in Fig. 5(e), its blue fluorescence is obviously burst and the characteristic emission of Eu located at $617 \mathrm{~nm}$ remains unchanged, so it shows the red color of Eu. The color change is obvious and can be used for the visual detection of $\mathrm{Fe}^{3+}$ by human eyes. In addition, this section analyzes and compares CD@Eu-MOF with $\mathrm{Fe}^{3+}$ fluorescent probes reported in other literatures, and the results are shown in the following Table 2. The results show that CD@Eu-MOF still has great advantages in comparison with other fluorescent probes.

Table 2 Comparison of Several Different Fe ${ }^{3+}$ Detection Methods

\begin{tabular}{|llll|}
\hline Fluorescence sensors & Detection limit $/ \mu \mathrm{M}$ & Linear range/ $\mu \mathrm{M}$ & Ref. \\
\hline GQDs & 7.22 & $1-80$ & {$[37]$} \\
\hline NCQDs & 4.67 & $1-50$ & {$[38]$} \\
\hline PPTA-AuNPs & 4.34 & $1-100$ & {$[39]$} \\
\hline Au nanoclusters & 3.5 & $5-1280$ & {$[40]$} \\
\hline Rhodamine B & 2.07 & $1-20$ & {$[41]$} \\
\hline CD@Eu-MOF & 0.91 & $1-200$ & This work \\
\hline
\end{tabular}

It was pointed out in a related paper that $\mathrm{Hg}^{2+}$ also has a certain burst effect on the citric acid carbon dots, so it will cause some interference to the detection of carbon dots on $\mathrm{Fe}^{3+}$, while CD@Eu-MOF 
circumvents this drawback well.[21] As shown in Fig.6(a), when CD@Eu-MOF is in $\mathrm{Hg}^{2+}$ solution, its red fluorescence completely disappears, leaving only the blue fluorescence of the carbon dots. analyzing the reason, it may be that $\mathrm{Hg}^{2+}$ will destroy the structure of the metal-organic framework and collapse its structure, thus Eu-MOF loses its red fluorescence. the XRD pattern of Fig. 6(b) also illustrates the problem: the $\mathrm{Fe}^{3+}$ treated CD@Eu-MOF still have a good crystal structure, while the XRD spectrum after $\mathrm{Hg}^{2+}$ treatment loses its characteristic peaks. Thus, the encapsulation of CD within Eu-MOF not only effectively improves the detection ability of $\mathrm{CD}$ for $\mathrm{Fe}^{3+}$, but also discriminates well for $\mathrm{Hg}^{2+}$, which can cause interference, with CD@Eu-MOF.In addition, the selectivity of CD@Eu-MOF for Fe ${ }^{3+}$ detection was also analyzed, and a series of metal ions that may interfere with CD@Eu-MOF were selected as interference terms. As shown in Fig. 5(c), the $I_{E U} / I_{C D}$ fluorescence intensity ratio in CD@Eu-MOF basically did not change much when other metal ions were added. As shown in Fig. 5(d), the color of CD@Eu-MOF is purple when no iron ion is added. And when iron ions are added, as shown in Fig. 5(e), its blue fluorescence is obviously burst and the characteristic emission of Eu located at $617 \mathrm{~nm}$ remains unchanged, so it shows the red color of Eu. The color change is obvious and can be used for the visual detection of $\mathrm{Fe}^{3+}$ by human eyes. In addition, this section analyzes and compares CD@Eu-MOF with Fe ${ }^{3+}$ fluorescent probes reported in other literatures, and the results are shown in the following Table 2. The results show that CD@Eu-MOF still has great advantages in comparison with other fluorescent probes.

\subsection{Fluorescent quenching mechanism}

Finally, the analysis of the fluorescence burst mechanism of CD@Eu-MOF by Fe ${ }^{3+}$ was carried out by Fig. 6(c). UV mapping. There is a strong absorption peak at $340 \mathrm{~nm}$, which may be caused by the energy jump captured from the ground state to the excited state on the surface of $C D$, and this is the reason for the strong fluorescence phenomenon of CD. When CD are loaded into Eu-MOF, a significant blue shift (325 $\mathrm{nm}$ ) of this peak can be found. For this phenomenon, it may be related to the synthesis of CD@Eu-MOF. In the synthesis precursor solution of CD@Eu-MOF, the solvent used is DMF, and CD are dissolved in DMF to participate in the hydrothermal reaction, while CD in DMF its UV absorption and fluorescence emission are significantly blue-shifted, as can be seen from the UV absorption of CD@Eu-MOF and CD in DMF. Both of them have obvious absorption peaks at $325 \mathrm{~nm}$. When CD@Eu-MOF is in contact with $\mathrm{Fe}^{3+}$, it can be seen that the absorption peak at $325 \mathrm{~nm}$ disappears. According to the $\mathrm{UV}$ absorption of $\mathrm{Fe}^{3+}$, it has no absorption peak in the $200-500 \mathrm{~nm}$ region, and the reason of competitive absorption can be excluded. Combined with the XRD plots of CD@Eu-MOF after reaction with $\mathrm{Fe}^{3+}$, etc., it is speculated that the reason is that $\mathrm{Fe}^{3+}$ complexes with groups such as $-\mathrm{COOH}$ on the surface of $\mathrm{CD}$, and the resulting complexes

burst the blue fluorescence of $\mathrm{CD}$ without interfering with the red fluorescence of Eu-MOF, which is the mechanism of the ratio metric fluorescence detection of $\mathrm{Fe}^{3+}$ by $\mathrm{CD} @ E \mathrm{E}-\mathrm{MOF}$.

\section{7 actual sample analysis}

In this section, in order to verify the detection performance of CD@Eu-MOF for $\mathrm{Fe}^{3+}$ in actual water samples, a series of experiments were carried out as described in 3.5, using Xinghu water of Wuhan 
University and tap water of the Department of Printing and Packaging as samples to detect $\mathrm{Fe}^{3+}$. The results are shown in the Table 3. The recoveries for tap water and Xinghu water are between 96.5\%-105.03\% and $98.63 \%-103.83 \%$, respectively, which proves that the CD@Eu-MOF material can actually detect the $\mathrm{Fe}^{3+}$ content in the samples.

Table 3 Detection of $\mathrm{Fe}^{3+}$ in real water samples by CD@Eu-MOF

\begin{tabular}{|lllll|}
\hline Sample & Add $\mathrm{Fe}^{3+} / \mu \mathrm{M}$ & Found $/ \mu \mathrm{M}$ & Recovery, $\%$ & $\mathrm{RSD}, \%$ \\
\hline Tap Water & 30 & 31.51 & $105.03 \%$ & 0.88 \\
\hline & 60 & 57.90 & $96.50 \%$ & 1.07 \\
\hline Xinghu Lake & 60 & 92.63 & $102.92 \%$ & 0.56 \\
\hline & 90 & 31.15 & $103.83 \%$ & 1.12 \\
\hline
\end{tabular}

\subsection{CD@Eu-MOF as fluorescent patterning agent}

In order to explore the practical application scenarios of the prepared CD@Eu-MOF, three fluorescent substances, CD, CD@Eu-MOF and Eu-MOF, were prepared into inks according to the methods shown in the literature.[42] The three prepared inks were printed into dot matrix by screen printing, and the results are shown in Fig. 6(d). It can be seen that it can still maintain a good fluorescence effect after printing, which provides the possibility to further expand the detection application of CD@Eu-MOF.

\section{Conclusions}

In summary, we formed CD@Eu-MOF by encapsulating CD and Eu ${ }^{3+}$ into MOF materials. the CD@Eu-MOF not only retained the fluorescence properties of $\mathrm{CD}$ and $\mathrm{Eu}^{3+}$, but also developed into an ultra-sensitive and highly selective $\mathrm{Fe}^{3+}$ sensor with a wide response range $(1-200 \mu \mathrm{M})$ and a very low detection limit of $0.91 \mu \mathrm{M}$. The composite has a sensitivity and detection capability for $\mathrm{Hg}^{2+}$. At the same time, we used the BP (Back Propagation) neural network recognized by computer vision to establish a database of the fluorescence RGB values of $\mathrm{CD}$ corresponding to the $\mathrm{Fe}^{3+}$ concentration. On the premise of greatly improving the recognition accuracy, the real-time detection of $\mathrm{Fe}^{3+}$ concentration by $\mathrm{CD}$ is also realized. The accuracy of the linear relationship between CD@Eu-MOF fluorescence spectrum and Fe ${ }^{3+}$ concentration was also verified by neural network. A feasible solution is provided for further simplifying the detection of $\mathrm{Fe}^{3+}$ in the environment.

\section{Declarations}


Supplementary Information The online version contains supplementary material available

Acknowledgements We acknowledge the usage of SEM, TEM, FTIR and XPS supported by the Open Subsidies for large-scale Equipment of Wuhan University (Grant No. LF20201282).

Author Contributions YZ: Methodology, Formal analysis, Writing - Review \& Editing. XW: Investigation, Writing - Original Draft. ZG: Data curation. XL: Conceptualization and Supervision. HL: Project administration. PZ: Validation. All authors read and approved the final manuscript.

Funding The National Natural Science Foundation of China (Grant No. 51776143) and Wuhan University postgraduate research credit course project of Intelligent Packaging and Food Safety (Grant No. 1506/413100017).

Data Availability All data generated or analyzed during this study are included in this published article.

Code Availability Not applicable.

Ethics Approval Not applicable.

Consent to Participate Not applicable.

Consent for Publication Not applicable.

Conflicts of Interest The authors declare that there is no competing financial interest or any other conflict of interest.

\section{References}

1. Han C, Wang R, Wang K, Xu H, Sui M, Li J, Xu K (2016) Highly fluorescent carbon dots as selective and sensitive "on-off-on" probes for iron(III) ion and apoferritin detection and imaging in living cells. Biosens Bioelectron 83: 229-236.10.1016/j.bios.2016.04.066

2. Gonzalez-Viveros N, Gomez-Gil P, Castro-Ramos J, Cerecedo-Nunez HH (2021) On the estimation of sugars concentrations using Raman spectroscopy and artificial neural networks. Food Chem 352.10.1016/j.foodchem.2021.129375

3. Shakya BR, Teppo H, Rieppo L (2021) Discrimination of melanoma cell lines with Fourier Transform Infrared (FTIR) spectroscopy. Spectrochim Acta a 254.10.1016/j.saa.2021.119665

4. Xiao H, Zhao S, Fan R, Hussain D, Wang X (2021) Simultaneous determination of short-chain fatty alcohols in aged oil and biodiesels by stable isotope labeling assisted liquid chromatography-mass spectrometry. Talanta 229.10.1016/j.talanta.2021.122223

5. Xu XY, Lian X, Hao JN, Zhang C, Yan B (2017) A double-stimuli-responsive fluorescent center for monitoring of food spoilage based on dye covalently modified EuMOFs: From sensory hydrogels to logic devices. Adv Mater 29(37): 1702298.10.1002/adma.201702298 
6. Desai ML, Jha S, Basu H, Singhal RK, Sharma PK, Kailasa SK (2018) Microwave-assisted synthesis of water-soluble $\mathrm{Eu}^{3+}$ hybrid carbon dots with enhanced fluorescence for the sensing of $\mathrm{Hg}^{2+}$ ions and imaging of fungal cells. New J Chem 42(8): 6125-6133.10.1039/C7NJ04835A

7. Liu G, Kong D, Han J, Zhou R, Gao Y, Wu Z, Zhao L, Wang C, Wang L, Lu G (2021) Solvent-controlled synthesis of full-color carbon dots and its application as a fluorescent food-tasting sensor for specific recognition of jujube species. Sensors and Actuators B: Chemical 342:129963.10.1016/j.snb.2021.129963

8. Qureashi A, Pandith AH, Bashir A, Malik LA (2021) Biomass-derived carbon quantum dots: A novel and sustainable fluorescent "ON-OFF-ON" sensor for ferric ions. Anal Methods 13(40): 47564766.10.1039/d1ay01112j

9. Guo Z, Huang X, Li Z, Shi J, Zhai X, Hu X, Zou X (2021) Employing CulnS2 quantum dots modified with vancomycin for detectingStaphylococcus aureus and iron(iii). Anal Methods-Uk 13(12): 15171526.10.1039/DOAY02253E

10. Baker SN, Baker GA (2010) Luminescent carbon nanodots: Emergent nanolights. Angewandte Chemie International Edition 49(38): 6726-6744.10.1002/anie.200906623

11. Lin X, Gao G, Zheng L, Chi Y, Chen G (2014) Encapsulation of strongly fluorescent carbon quantum dots in metal-organic frameworks for enhancing chemical sensing. Anal Chem 86(2): 12231228.10.1021/ac403536a

12. Hou X, Song J, Wu Q, Lv H (2021) Chiral carbon quantum dots as fluorescent probe for rapid chiral recognition of isoleucine enantiomers. Anal Chim Acta 1184.10.1016/j.aca.2021.339012

13. Hu Y, Gao Z, Luo J (2021) Fluorescence detection of malachite green in fish tissue using red emissive Se,N,Cl-doped carbon dots. Food Chem 335.10.1016/j.foodchem.2020.127677

14. Hu X, Li Y, Xu Y, Gan Z, Zou X, Shi J, Huang X, Li Z, Li Y (2021) Green one-step synthesis of carbon quantum dots from orange peel for fluorescent detection of Escherichia coli in milk. Food Chem 339.10.1016/j.foodchem.2020.127775

15. Wang Y, Han C, Yu L, Wu J, Min Y, Tan J, Zhao Y, Zhang P (2020) Etching-controlled suppression of fluorescence resonance energy transfer between nitrogen-doped carbon dots and Ag nanoprisms for glucose assay and diabetes diagnosis. Spectrochim Acta a 242.10.1016/j.saa.2020.118713

16. Jalili R, Khataee A, Rashidi M, Razmjou A (2020) Detection of penicillin G residues in milk based on dual-emission carbon dots and molecularly imprinted polymers. Food Chem

314.10.1016/j.foodchem.2020.126172

17. Lee G, Munir S, Park S (2018) Label-Free Detection of Dopamine based on Photoluminescence of Boronic Acid-Functionalized Carbon Dots in Solid-State Polyethylene Glycol Thin Film. Macromol Res 26(12SI): 1150-1159.10.1007/s13233-019-7025-4

18. Ahmed F, Iqbal S, Zhao L, Xiong H (2021) "ON-OFF-ON" fluorescence switches based on N,S-doped carbon dots: Facile hydrothermal growth, selective detection of $\mathrm{Hg}^{2+}$, and as a reversive probe for guanine. Anal Chim Acta 1183.10.1016/j.aca.2021.338977 
19. Wang Y, Hu X, Li W, Huang X, Li Z, Zhang W, Zhang X, Zou X, Shi J (2020) Preparation of boron nitrogen co-doped carbon quantum dots for rapid detection of $\mathrm{Cr}(\mathrm{VI})$. Spectrochim Acta a 243.10.1016/j.saa.2020.118807

20. Liao S, Zhao X, Zhu F, Chen M, Wu Z, Song X, Yang H, Chen X (2018) Novel S, N-doped carbon quantum dot-based "off-on" fluorescent sensor for silver ion and cysteine. Talanta 180: 300308.10.1016/j.talanta.2017.12.040

21. Xu X, Yan B (2016) Fabrication and application of a ratiometric and colorimetric fluorescent probe for $\mathrm{Hg}^{2+}$ based on dual-emissive metal-organic framework hybrids with carbon dots and $\mathrm{Eu}^{3+}$. J Mater Chem C 4(7): 1543-1549.10.1039/c5tc04002g

22. Qin S, Yan B (2018) Dual-emissive ratiometric fluorescent probe based on Eu ${ }^{3+} / \mathrm{C}$-dots@MOF hybrids for the biomarker diaminotoluene sensing. Sensors and Actuators B: Chemical 272: 510517.10.1016/j.snb.2018.06.018

23. Jia R, Tian W, Bai H, Zhang J, Wang S, Zhang J (2019) Amine-responsive cellulose-based ratiometric fluorescent materials for real-time and visual detection of shrimp and crab freshness. Nat Commun. 10.10.1038/s41467-019-08675-3

24. Yuan C, Qin X, Xu Y, Shi R, Cheng S, Wang Y (2021) Dual-signal uric acid sensing based on carbon quantum dots and o-phenylenediamine. Spectrochim Acta a 254.10.1016/j.saa.2021.119678

25. Chen J, Jiang S, Wang M, Xie X, Su X (2021) Self-assembled dual-emissive nanoprobe with metalorganic frameworks as scaffolds for enhanced ascorbic acid and ascorbate oxidase sensing. Sensor Actuat B-Chem 339.10.1016/j.snb.2021.129910

26. Li Y, He Y, Ge Y, Song G, Zhou J (2021) Smartphone-assisted visual ratio-fluorescence detection of hypochlorite based on copper nanoclusters. Spectrochim Acta a 255.10.1016/j.saa.2021.119740

27. Hao A, Wang X, Mei Y, Nie J, Yang Y, Dai C (2021) A smartphone-combined ratiometric fluorescence probe for specifically and visibly detecting cephalexin. Spectrochim Acta a 249.10.1016/j.saa.2020.119310

28. Sun J, Lu Y, He L, Pang J, Yang F, Liu Y (2019) A colorimetric sensor array for protein discrimination based on carbon nanodots-induced reversible aggregation of AuNP with GSH as a regulator. Sensor Actuat B-Chem 296.10.1016/j.snb.2019.126677

29. Zhou L, Zhou P, Zhang Y, Liu B, Gao P, Guo S (2021) 3D star-like atypical hybrid MOF derived singleatom catalyst boosts oxygen reduction catalysis. J Energy Chem 55: 355360.10.1016/j.jechem.2020.06.059

30. Guan L, Jiang Z, Cui Y, Yang Y, Yang D, Qian G (2021) An MOF-Based luminescent sensor array for pattern recognition and quantification of metal ions. Adv Opt Mater 9(9).10.1002/adom.202002180

31. Bonneau M, Lavenn C, Ginet P, Otake K, Kitagawa S (2020) Upscale synthesis of a binary pillared layered MOF for hydrocarbon gas storage and separation. Green Chem 22(3): 718-

724.10.1039/c9gc03561c 
32. Qin S, Hao J, Xu X, Lian X, Yan B (2017) Highly sensing probe for biological metabolite of benzene series pollutants based on recyclable $\mathrm{Eu}^{3+}$ functionalized metal-organic frameworks hybrids. Sensors and Actuators B: Chemical 253: 852-859.10.1016/j.snb.2017.07.020

33. Liu C, Luo Y, Wen H, Qi Y, Shi G, Deng J, Zhou T (2021) Red-to-blue paper-based colorimetric sensor integrated with smartphone for point-of-use analysis of cerebral AChE upon $\mathrm{Cd}^{2+}$ exposure. Nanoscale 13(2): 1283-1290.10.1039/d0nr07449g

34. Fernandez-Canque H, Hintea S, Csipkes G, Pellow A, Smith H (2008) Machine Vision application to the detection of micro-organism in drinking water. KNOWLEDGE-BASED INTELLIGENT INFORMATION AND ENGINEERING SYSTEMS, PT 3, PROCEEDINGS 5179: 302

35. Yan B (2021) Luminescence response mode and chemical sensing mechanism for lanthanidefunctionalized metal-organic framework hybrids. Inorg Chem Front 8(1): 21-

233.10.1039/d0qi01153c

36. Thiyagarajan SK, Raghupathy S, Palanivel D, Raji K, Ramamurthy P (2016) Fluorescent carbon nano dots from lignite: Unveiling the impeccable evidence for quantum confinement. Phys Chem Phys 18(17): 12065-12073.10.1039/c6cp00867d

37. Ananthanarayanan A, Wang X, Routh P, Sana B, Lim S, Kim D, Lim K, Li J, Chen P (2014) Facile synthesis of graphene quantum dots from $3 \mathrm{D}$ graphene and their application for $\mathrm{Fe}^{3+}$ sensing. Adv Funct Mater 24(20): 3021-3026.10.1002/adfm.201303441

38. Yu J, Xu C, Tian Z, Lin Y, Shi Z (2016) Facilely synthesized N-doped carbon quantum dots with high fluorescent yield for sensing $\mathrm{Fe}^{3+}$. New J Chem 4(3): 283-288.10.1039/c5nj03252k

39. Anwar A, Minhaz A, Hussain SS, Anwar A, Simjee SU, Ishaq M, Khan NA, Shah MR (2019) Pyrazinium thioacetate capped gold nanoparticles as $\mathrm{Fe}$ (III) sensor and Fe(III) marked anti-proliferating agent in human neuroblastoma cells. Spectrochimica Acta Part A: Molecular and Biomolecular Spectroscopy 206: 135-140.10.1016/j.saa.2018.07.099

40. Annie Ho J, Chang H, Su W (2012) DOPA-Mediated reduction allows the facile synthesis of fluorescent gold nanoclusters for use as sensing probes for ferric ions. Anal Chem 84(7): 32463253.10.1021/ac203362g

41. Li H, Liu Z, Jia R (2021) "Turn-on" fluorescent probes based on Rhodamine B/amino acid derivatives for detection of $\mathrm{Fe}^{3+}$ in water. Spectrochimica Acta Part A: Molecular and Biomolecular Spectroscopy 247: 119095.10.1016/j.saa.2020.119095

42. Fu X, Li G, Cai S, Yang H, Lin K, He M, Wen J, Li H, Xiong Y, Chen D, Liu X (2021) Color-switchable hybrid dots/hydroxyethyl cellulose ink for anti-counterfeiting applications. Carbohyd Polym 251: 117084.10.1016/j.carbpol.2020.117084

\section{Figures}


a
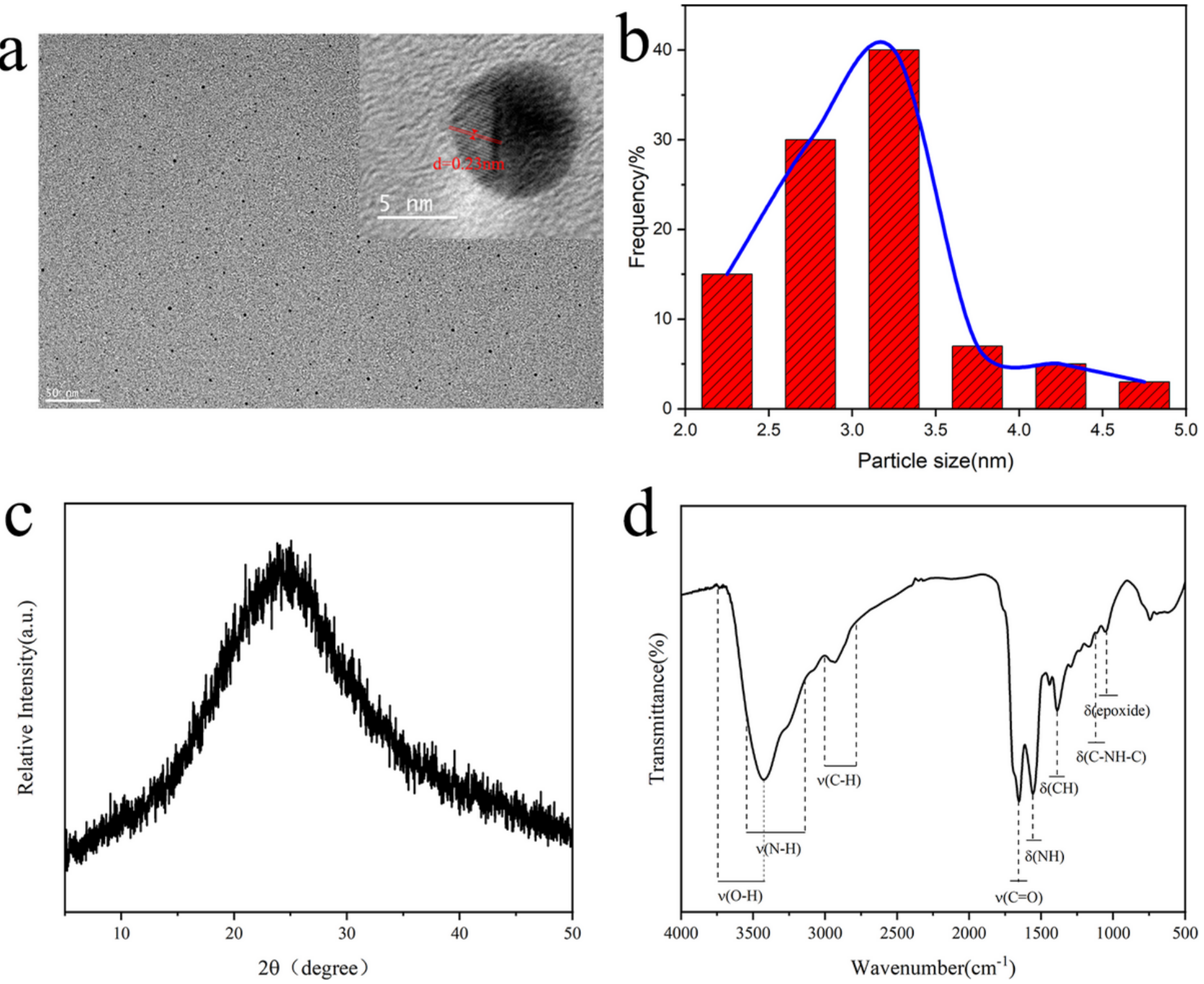

Figure 1

(a) TEM image of as-prepared CD; (b) Particle size distribution map of as-prepared CD; (c) XRD patterns of as-prepared CD; (d) Fourier transform infrared spectrogram of as-prepared CD. 

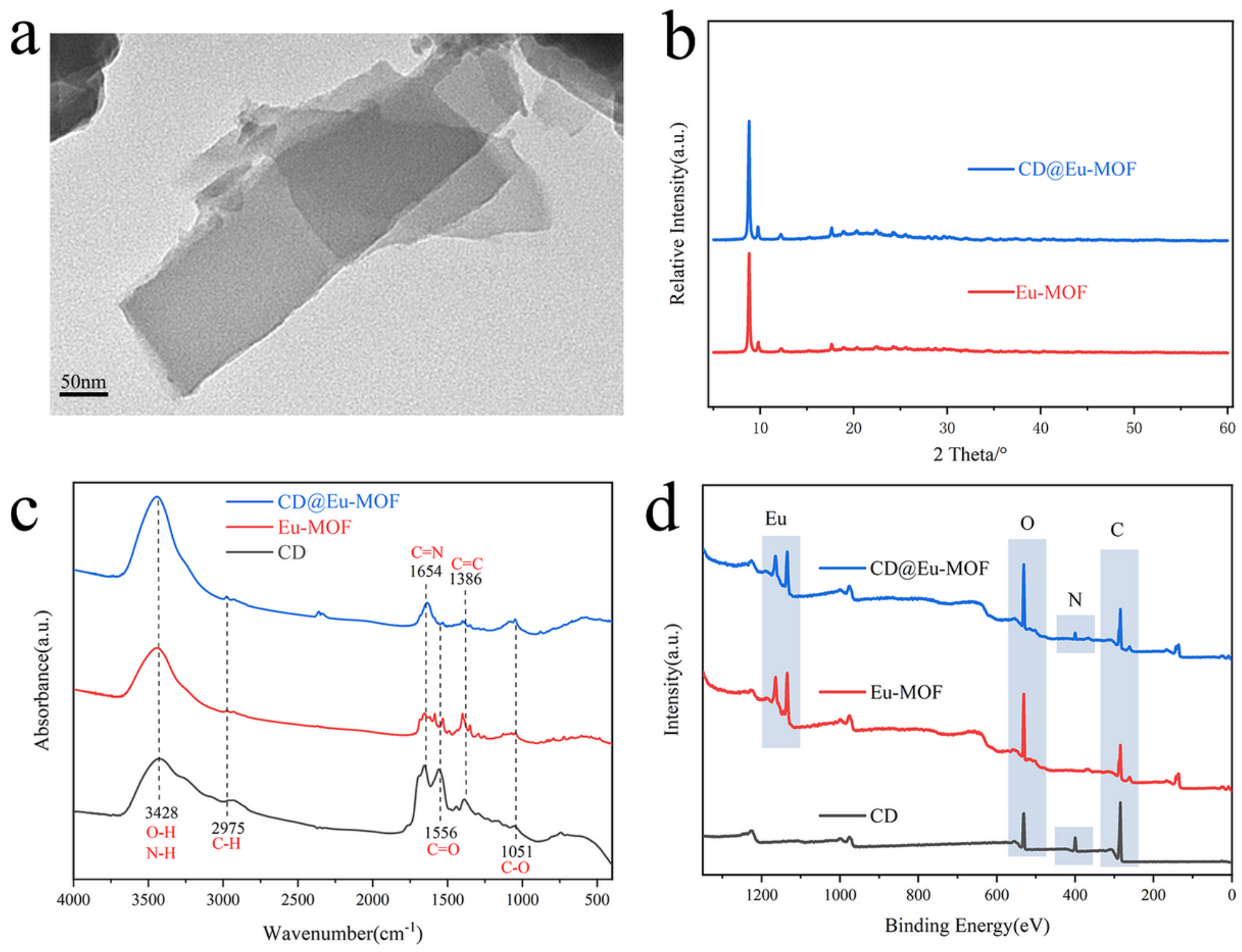

\section{Figure 2}

(a) TEM image of as-prepared CD@Eu-MOF; (b) XRD patterns of as-prepared Eu-MOF and CD@Eu-MOF;

(c) Infrared spectrogram of as-prepared CD, Eu-MOF and CD@Eu-MOF; (d) The XPS spectra of CD, EuMOF and CD@Eu-MOF. 

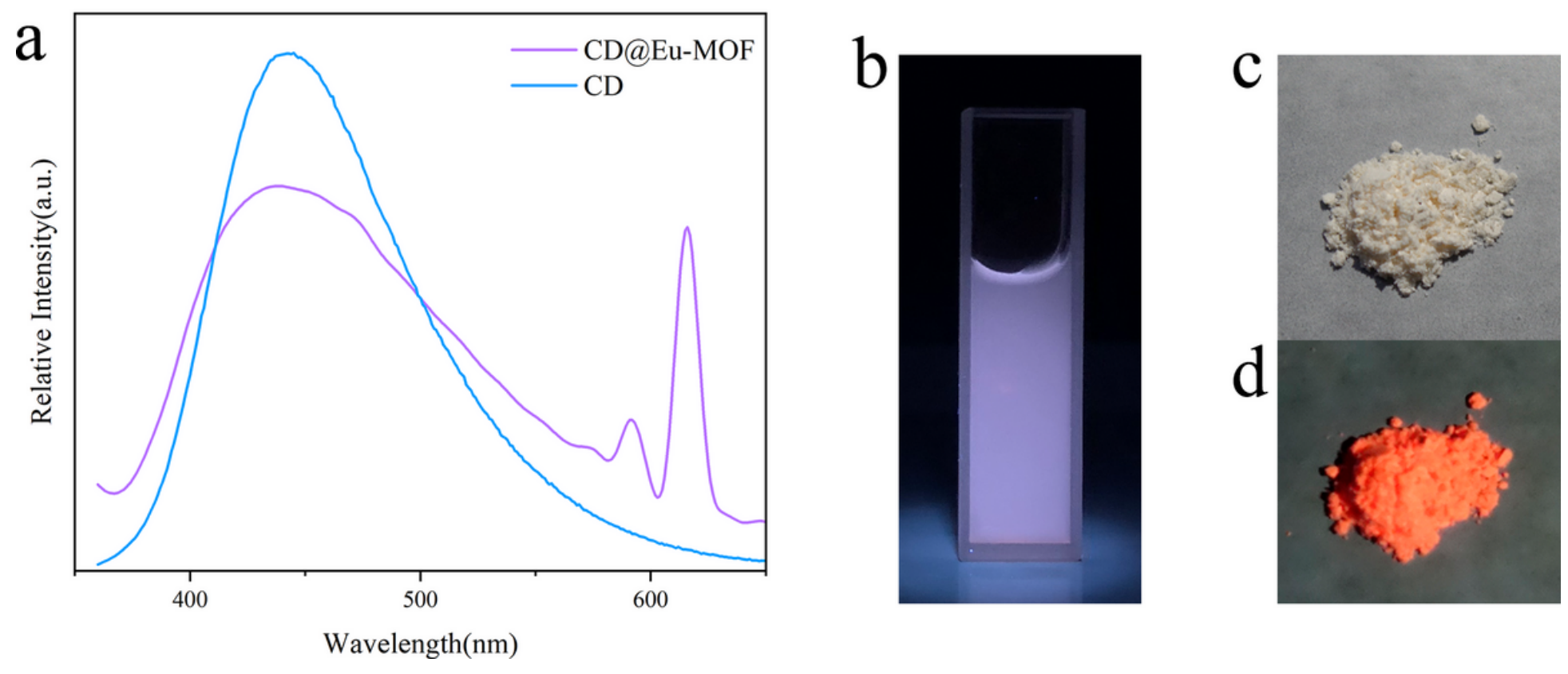

\section{Figure 3}

(a) Fluorogram of as-prepared CD and CD@Eu-MOF; (b) Solution of as-prepared CD@Eu-MOF under UV light irradiation at $365 \mathrm{~nm}$; (c) Powder of as-prepared Eu-MOF under visible light; (d) UV light irradiation at $365 \mathrm{~nm}$. 

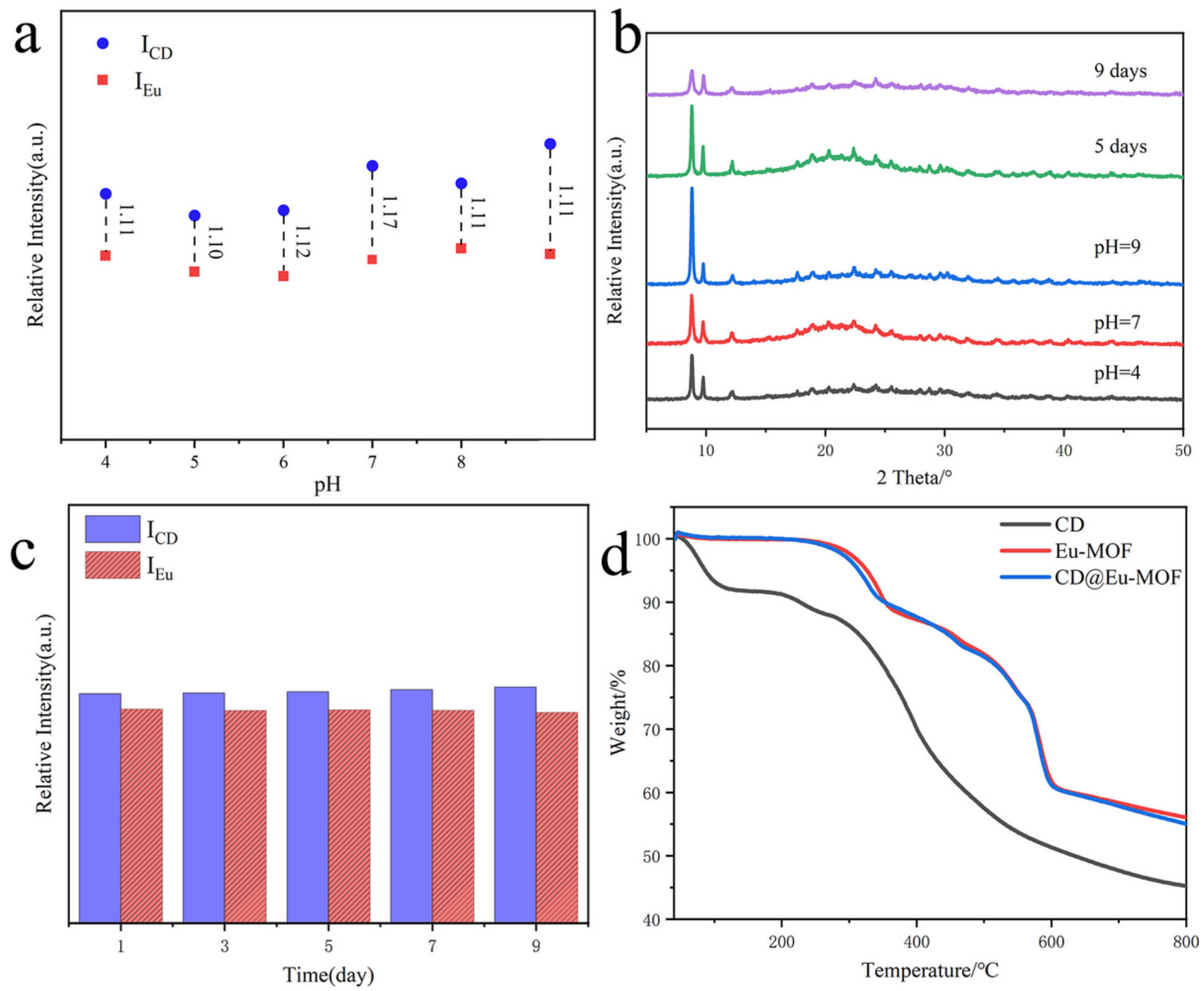

Figure 4

(a) Stability of PL intensity of CD@Eu-MOF after immersing in different $\mathrm{pH}$ aqueous solutions for $1 \mathrm{~h}$; (b) XRD patterns of CD@Eu-MOF after exposure to different $\mathrm{pH}$ and different storage time in $\mathrm{H}_{2} \mathrm{O}$; (c) Stability of PL intensity of CD@Eu-MOF after treated in aqueous solution for 9 days; (d) Thermogravimetry of CD, Eu-MOF, CD@Eu-MOF. 

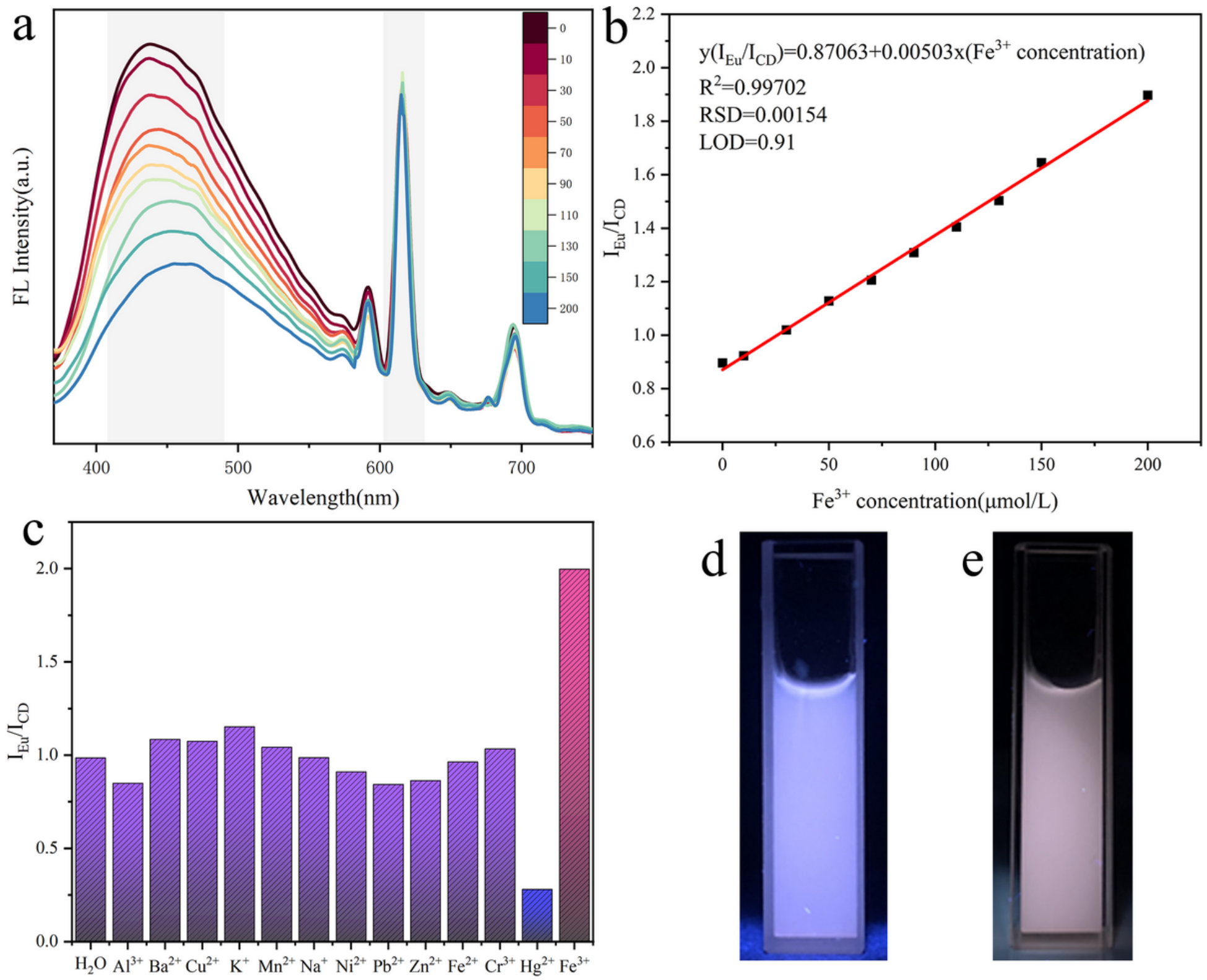

\section{Figure 5}

(a)Fluorogram of as-prepared CD and CD@Eu-MOF at different concentrations of $\mathrm{Fe}^{3+}$; (b) Linear relationship between $I_{E U} / I_{C D}$ and $\mathrm{C}_{\mathrm{Fe}}{ }^{3+}$ and inset shows images of the test plates treated by different concentration of $\mathrm{Fe}^{3+}$; (c) The change of $I_{E u} / I_{C D}$ under different metal ions; Solution of as-prepared CD@Eu-MOF under UV light irradiation at $365 \mathrm{~nm}$ without $\mathrm{Fe}^{3+}(\mathrm{d})$ and with $\mathrm{Fe}^{3+}(\mathrm{e})$. 

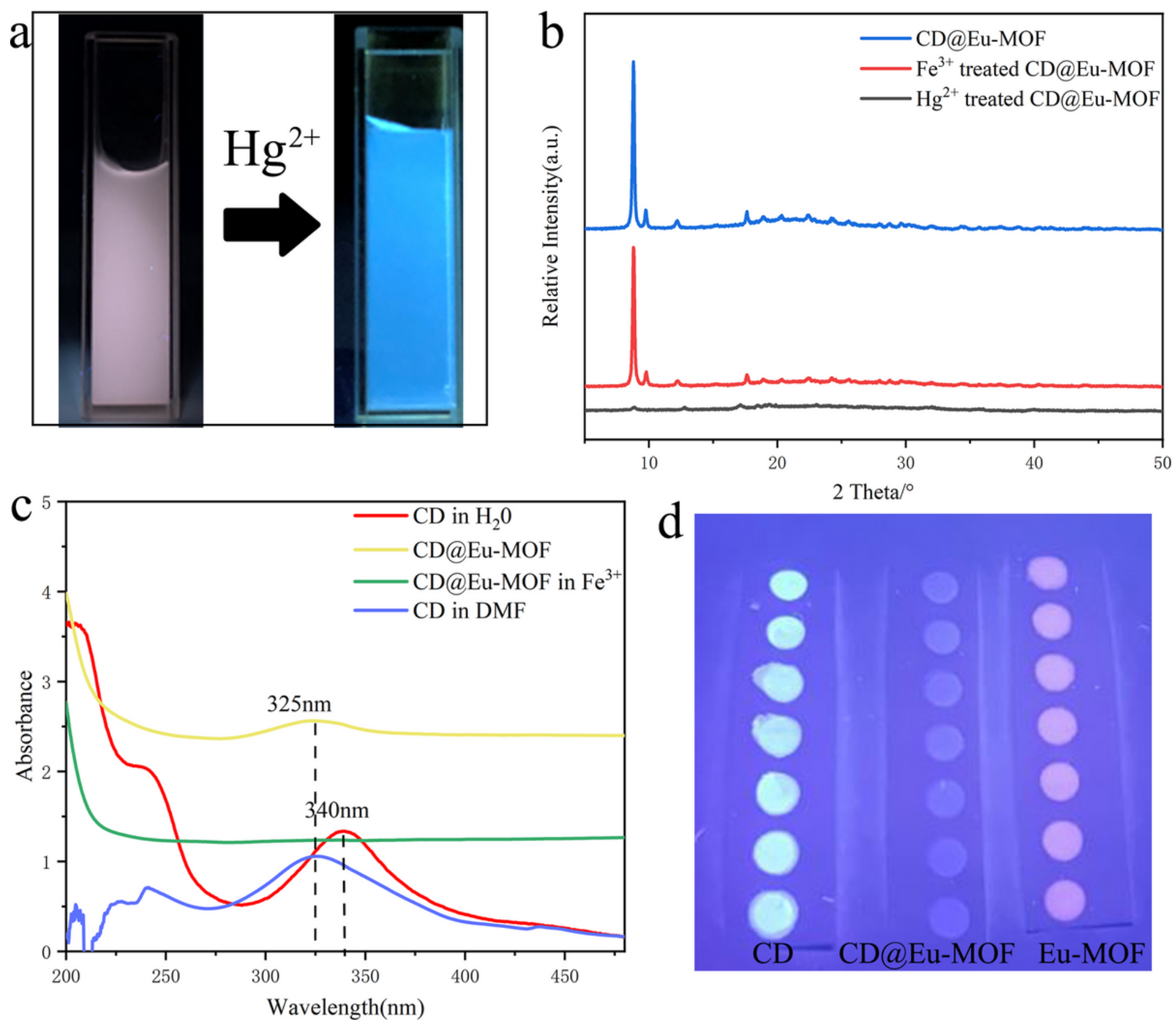

\section{Figure 6}

(a) Solution of as-prepared CD@Eu-MOF under UV light irradiation at $365 \mathrm{~nm}$ (The left side is without $\mathrm{Hg}^{2+}$, and the right side is with $\mathrm{Hg}^{2+}$ ); (b) XRD patterns of CD@Eu-MOF, Fe ${ }^{3+}$ treated CD@Eu-MOF and $\mathrm{Hg}^{2+}$ treated CD@Eu-MOF; (c) UV-spectrum of CD in $\mathrm{H}_{2} \mathrm{O}$, CD@Eu-MOF, CD@Eu-MOF in Fe ${ }^{3+}$ and CD in DMF; (d) Fluorescent lattice images drawn using CD, CD@Eu-MOF and Eu-MOF based fluorescent inks.

\section{Supplementary Files}

This is a list of supplementary files associated with this preprint. Click to download.

- SupportInformation.docx 